Background Tumour cells often exhibit altered energy metabolism for tumour progression, but whether long noncoding RNAs (lncRNAs) are involved in this process remains elusive. We aimed to identify novel lncRNAs significantly affecting the development of Esophageal Squamous Cell Carcinoma (ESCC) and investigate the metabolism-related mechanisms.

Methods A siRNA library was established using top 100 lncRNAs highly expressed in ESCC screened from TCGA database and transfected into ESCC cells to identify lncRNAs that significantly affected cell glucose metabolism and proliferation. RNAscope in situ hybridization, qRT-PCR and RNAi assays were performed to investigate the functional role of lncRNA AGPG (Actin Gamma 1 Pseudogene) and clinical relevance. In vivo, cell-based and patient-derived xenograft (PDX) models were used to further explore roles of AGPG in ESCC tumorigenesis. RNA pull-down, mass spectrometry analyses, western blot and RNA-binding protein immunoprecipitation (RIP) were performed to identify interaction proteins and related mechanisms.

Results We identified that lncRNA AGPG was markedly increased in ESCC and correlated with poor prognosis. AGPG depletion significantly repressed ESCC cell proliferation and glycolytic activity including decreased glucose uptake and increased lactate production. In vivo experiments further showed that silencing $A G P G$ decreased tumours growth in cell-based xenografts and PDX models. Mechanistically, AGPG could interact with 6-phosphofructo-2-kinase/fructose-2, 6biphosphatase 3 (PFKFB3) in the nucleus, thus enhancing PFKFB3 stability via preventing its ubiquitination and proteosomal degradation. Subsequently, AGPG-PFKFB3 promoted glycolysis and dysregulated cell cycle by suppressing p27 and over-activating cyclin-dependent kinase 1(CDK1), thereby leading to G1/S cell cycle progression. Additionally, TP53 could bind to the promoter of $A G P G$ to negatively regulate its expression.

Conclusions We first identified that $A G P G$, which is regulated by TP53, is playing a pivotal role in glucose metabolism remodelling and cell proliferation through enhancing PFKFB3 stability, thus facilitating the development of ESCC. Collectively, our study suggests that TP53-AGPG-PFKFB3 axis might serve as potential biomarkers and therapeutic targets in ESCC.

\section{IDDF2018-ABS-0191 ABERRANT GUT MUCOSAL MICROBIOME SIGNATURES OF MALAYSIAN COLORECTAL CANCER PATIENTS}

${ }^{1}$ Muhammad Afiq Osman*, 'Hui-min Neoh, ${ }^{1}$ Nurul Syakima Ab Mutalib, ${ }^{1}$ Siok Fong Chin, ${ }^{2}$ Luqman Mazlan, ${ }^{2}$ Andee Dzulkarnaen Zakaria, ${ }^{3}$ Raja Affendi Raja Ali, ${ }^{3}$ Chai Soon Ngiu, ${ }^{1}$ Mia Yang Ang, 'Rahman Jamal. 'UKM Medical Molecular Biology Institute, Universiti Kebangsaan Malaysia, Kuala Lumpur, Malaysia; '2Department of Surgery, Faculty of Medicine, Universiti Kebangsaan Malaysia, Kuala Lumpur, Malaysia; ${ }^{3}$ Department of Medicine, Faculty of Medicine, Universiti Kebangsaan Malaysia, Kuala Lumpur, Malaysia

\subsection{6/gutjnl-2018-IDDFabstracts.24}

Background The human gut holds the densest microbiome ecosystem essential in maintaining healthy host physiology, whereby disruption of this ecosystem has been linked to the development of colorectal cancer (CRC). Accordingly, 16S rRNA sequencing studies on CRC gut mucosal tissues have found bacteria such as Fusobacterium nucleatum and Bacteroides fragilis to be over-represented in CRC patients from various regions in the world. As CRC is one of the most common malignancies in Malaysia, this study was carried out to profile the gut microbiome and identify over-represented bacteria in Malaysian CRC patients.

Methods This study was carried out from 2014 till 2017, where a total of $18 \mathrm{CRC}$ patients and 18 healthy controls from UKM Medical Centre, Kuala Lumpur, Malaysia were included. 16S rRNA sequencing of gut mucosal tissue samples targeting the 16S rRNA V3/V4 regions was performed with an Illumina $\mathrm{MiSeq}(\mathrm{R})$ sequencer, while diversity analyses were carried out using QIIME to determine differences between gut mucosal microbiome profile of CRC patients compared to healthy controls.

Results We observed a significant difference in gut mucosal microbiome composition of CRC patients compared to healthy controls. Higher relative abundance of Fusobacteria and Verrucomicrobia phyla were detected. Further linear discriminant analysis (LDA) effect size analysis showed significant enrichment of Fusobacterium, Parvimonas, Akkermansia and Peptostreptococcus genera in CRC patients. Interestingly, these bacterial genera were found to be enriched in all CRC cases of this study, regardless of patient demographics and CRC staging.

Conclusions In summary, we successfully characterised the gut mucosa-associated microbiome of Malaysian CRC patients. Significant abundance of Fusobacterium, Parvimonas, Akkermansia, and Peptostreptococcus in these patients indicates the importance of these genera towards colorectal carcinogenesis. Nevertheless, a larger study to test the reproducibility of these results will be crucial before these bacteria could be utilised as screening biomarkers for CRC in Malaysians.

\section{IDDF2018-ABS-0193 CYTOKINE IL9 MEDIATES THE PATHOGENESIS OF CROHN'S DISEASE THROUGH THE MIR21-CLDN8 PATHWAY}

Shenghong Zhang*, Li Li, Shanshan Huang, Ting Feng, Gaoshi Zhou, Minhu Chen. Department of Gastroenterology, The First Affiliated Hospital, Sun Yat-sen University, Guangzhou, China

\subsection{6/gutjnl-2018-IDDFabstracts.25}

Background Cytokine interleukin-9 (IL9) plays an essential role in the pathogenesis of inflammatory bowel disease. However, the molecular mechanism underlying the IL9 pathway remains unknown. Here, we initiate a series of studies to characterise the essential components of this pathway.

Methods The expression of IL9 in colon biopsies from Crohn's disease (CD) and controls were examined by Western blot and immunohistochemistry. The trinitrobenzene sulfonic acid (TNBS)-induced colitis model was used to verify the therapeutic efficiency of anti-IL9 mAb. Bioinformatics analysis was performed to predict putative candidate microRNAs that mediate the crosstalk between the IL9 proinflammatory signal and the downstream target gene in intestinal barrier function. Caco-2, NCM460 and SW480 cells were used to assess the specific pathway in vitro.

Results We demonstrated the proinflammatory role of IL9 in colonic mucosa of patients with Crohn's disease (CD). Animals treated with anti-IL9 exhibited significant recovery of many cardinal signs of colitis. The junction complex protein Claudin 8 (CLDN8) was identified as a critical downstream component of the IL9 inflammatory cascade. Anti-IL9 treatment alleviated TNBS-induced colitis by restoring CLDN8 levels in colonic 